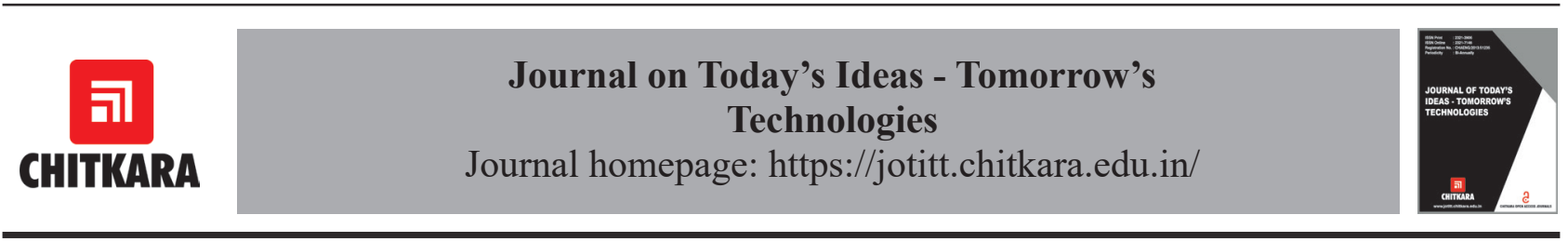

\title{
Antenna Design and Fabrication with Circular Microstrip Patch: A Review
}

\author{
R.V. Shinde, B. Gabane, T. H. Mujawar, and M. S. Kasbe* \\ Solapur University, Solapur-413255, Maharashtra, India \\ *kasbems@gmail.com (Corresponding Author)
}

\section{ARTICLE INFORMATION}

Received: August 31, 2020

Revised: October 26, 2020

Accepted: November 18, 2020

Published Online: June 22, 2021

Keywords:

Circular MSP, CST MWS,

Radiation pattern, Return loss,

VNA, Voltage Standing Wave

Ratio

\begin{abstract}
The paper presents a review on antenna designing and fabrication techniques with Microstrip Patch (MSP) development. The requirements of MSP are remote controlling, mobile communication, space communication and all wireless communication areas. Aim of this development is to provide a new approach of the designing and simulation of circular MSP for $5.8 \mathrm{GHz}$ operating frequency. CST MICROWAVE STUDIO (MWS) facilitates the quick and accurate analysis of high frequency $(\mathrm{HF})$ antennas which gives the responses and results before fabrication of actual patch with complete technology for 3D EM. FR-4 glass epoxy material board is used for fabrication which is admired and adaptable highpressure thermoset plastic laminate grade with superior strength to weight ratios. Circular MSP is easy to fabricate, portable, lightweight and low cost antenna. N9923A Field Fox RF VNA (Vector Network Analyser) were used for studying and testing all the parameters of fabricated MSP
\end{abstract}

\section{Introduction}

The wireless techniques diminished the complications and the limitations of wired techniques. Most of the common applications of wireless or modern wireless technology such as Wi-Fi, (Long Term Evolution) LTE, LTE-Advanced and Bluetooth [1, 2] are developed during 2000s. Moreover, applications of wireless communication are mobile communication (CellularGlobal System for Mobile Communications- GSM, CDMA, LTE), satellite communication, Wireless Local Area Network (WLAN), Wireless Personal Area Network WPAN (Zigbee) and Infrared [3]. Antenna is the heart of wireless communication. Antenna designing and fabrication is an active and fascinating concept for researchers and scientists. The compact, cost effective, portable and lightweight antennas are the requirements in the modern technology and wireless communication systems. Many types and shapes of antennas are introduced as per convenience and applications such as wire, aperture, reflector, lens, microstrip, array etc..

MSP antennas have many applications because of fabrication process, small size and shapes, portable and light weight. Many techniques have been applied to design, simulation and fabrication of a patch antenna. MSP antennas are named based on the profile of the radiating patch. MSP classified by the shapes and polarization types. It can be designed for linear, circular, elliptical polarization with respect to polarization techniques. Also the metal layer is available and simulate in the shapes such as circular, triangular, elliptical, square, ring sector, circular ring rectangular patch which are easy 
to design and analyze all the parameters [4]. The Circular MSP is simple to design and development compared with rectangular, triangular MSP because of the geometry of circle, which has one degree of freedom i. e. radius. Therefore, circular MSP (C-MSP) is simpler to design, simulation and the size is reduced up to $16 \%$ compared with rectangular patch for same frequency [5-6].

The microstrip antenna with conducting patch was developed in 1970. Nowadays, low cost metal plane and little strip is the choice of designers and researchers for radiation of the signals [7]. MSP is the greater part of the necessities for mobile, satellite equipment and numerous business requirements are satisfied by the utilization of it [8]. Radar is one of the applications where the UWB microstrip is used the most commonly used because of their advantages like- ease of fabrication simple structure, easy integration with microwave integrated circuits and geometric shape of a microstrip antenna [9-11]. In case of MSP the improvement of bandwidth is achieved by loading a different shape and size of slots and notches on the patch or in the ground plane [12]. Today's large amounts of software's are pioneer for antenna design, analysis and simulation. Microwave studio CST [13], Ansoft HFSS (high-frequency structure simulator) [14, 15], Integral Equation Three-Dimensional (electromagnetics) IE3D, FEKO, antenna Magus like computer tools are used to design and simulation of an antenna. Also MATLAB (Matrix Laboratory) and simulink environment and programming language provide many functions, tools and apps for the antenna design, analysis and visualization. All of these having its own specifications, features and function tools which are depending on the antenna elements, geometry, parameters (Radiation patterns, Directivity, Antenna gain, Efficiency, Beamwidth, Bandwidth, Smith chart, frequency ranges etc.) and requirements. Out of those some software's having limitations like speed, frequency ranges, geometry, accuracy etc..

In the fabrication process of patch antenna (or PCB type antenna) designing and fabrication many techniques are introduced like Inkjet printing technology [16], Screen printing, Flexography method, Chemical Etching, Computer Controlled Coordinatograph method etc. Present paper gives the information about development of MSP for $5.8 \mathrm{GHz}$ with design, simulation and fabrication. Moreover, avoiding blocking in the low frequency ISM band $(2.45 \mathrm{GHz})$, using the $5.8 \mathrm{GHz}$ band allows for higher data rates. Some of the products that specifically make use of the $5.8 \mathrm{GHz}$ band are baby monitors, cordless phones and cameras. In the RFID technology research $5.8 \mathrm{GHz}$ ISM band is being carried out also.

In the process of antenna design and fabrication important part is requirement, which is depending on the parameters of the antenna. High directivity and narrow beamwidth of an antenna are the common requirements for wireless power transmission (WPT) systems [17].

\section{Methodology}

\subsection{Designing and Specifications of Circular MSP}

A Circular MSP antenna is designed at the frequency of 5.8 GHz and CST MWS tool with CST MICROWAVE STUDIO software is used to design and simulate antenna before fabrication. Fig. 1 shows the geometry of circular MSP with coordinates (x, y).

1. Dimensions and specification

Dielectric constant $\left(\varepsilon_{\mathrm{r}}\right)=4.3$ (for the FR4 substrate)

Height of dielectric substrate $(\mathrm{h})=1.6 \mathrm{~mm}$

Resonant frequency (fr) $=5.8 \mathrm{GHz}$

Velocity of light in vacuum $(\mathrm{c})=3.8 \times 10^{8}$

Height of conductor $(\mathrm{ht})=0.035 \mathrm{~mm}$

Circular patch radius is calculated by using the below formula.

$$
\begin{aligned}
& a=\frac{F}{\left\{1+\frac{2 h}{\pi F \varepsilon_{r}}\left[\ln \left(\frac{\pi F}{2 h}\right)+1.7726\right]\right\}^{1 / 2}} \\
& F=\frac{8.791 \times 10^{9}}{f_{\mathrm{r}} \sqrt{\varepsilon_{r}}}
\end{aligned}
$$

Hence radius of the patch is $7.05 \mathrm{~mm}$

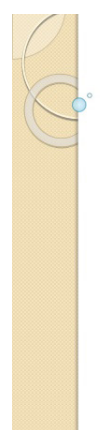

\section{Circular patch}

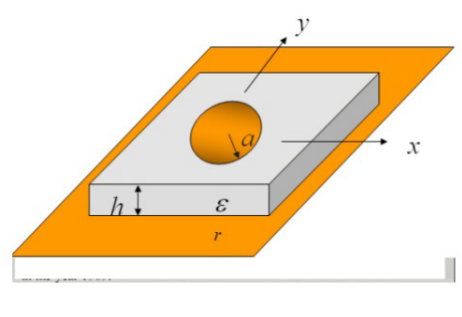

Figure 1: Geometry of circular MSP

\subsection{Flow of Designing and Simulation in CST} Software

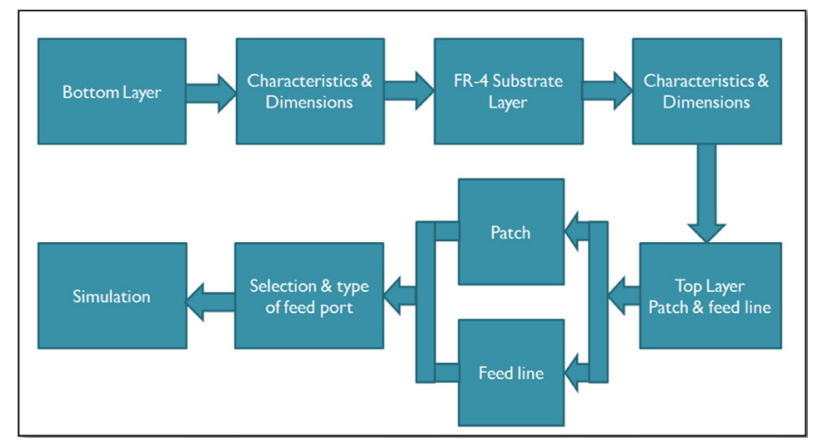

Figure 2: Patch designing flow with CST 


\subsection{Fabrication of an Antenna}

After the designing and simulation an antenna is fabricated on FR4 PCB. There are 5 steps of fabrication process.

The first step of fabrication process is transparency film, in which the EM structure is transfer from MOS (Microwave Office Software) to the Auto-computer Added Drawing (i.e. AutoCAD) and then print it onto the transparency film. Then the image circuit pattern is transfer under the Ultraviolet exposure process. In this process an image of circuit or patch is transfer on the photo resist laminated board in the UV exposure machine. The UV or photo exposure process is completed within 2 minutes. Third is the developing process, in which exposed resist wash away by using photo resist developer solution. 10 minutes are required for this process. After that this solution is removed using the spray wash. Water and Sodium Hydroxide $(\mathrm{NaOH})$ is used for spray wash. After that unwanted copper or metal are removed under etching process. Ferric chloride is used to clean copper from the base or FR4 substrate layer. This process took about 30-50 minutes to be done which is depending on the dilution of the ferric chloride. The final step is to soldering in which the SMA connector is connected to the feed line. The board is dried and rubbed using sand paper to clean the remaining unexposed resist pattern area. In the lead fabrication, the SMA connector was soldered to the MSP antenna. The flow of this fabrication process is shown in Fig. 3. The top and bottom view of patch after fabrication is shown in Fig. 4.

Once fabrication process is completed, the response of an antenna is tested with N9923A FieldFox RF VNA (Vector Network Analyzer) for higher frequency application.

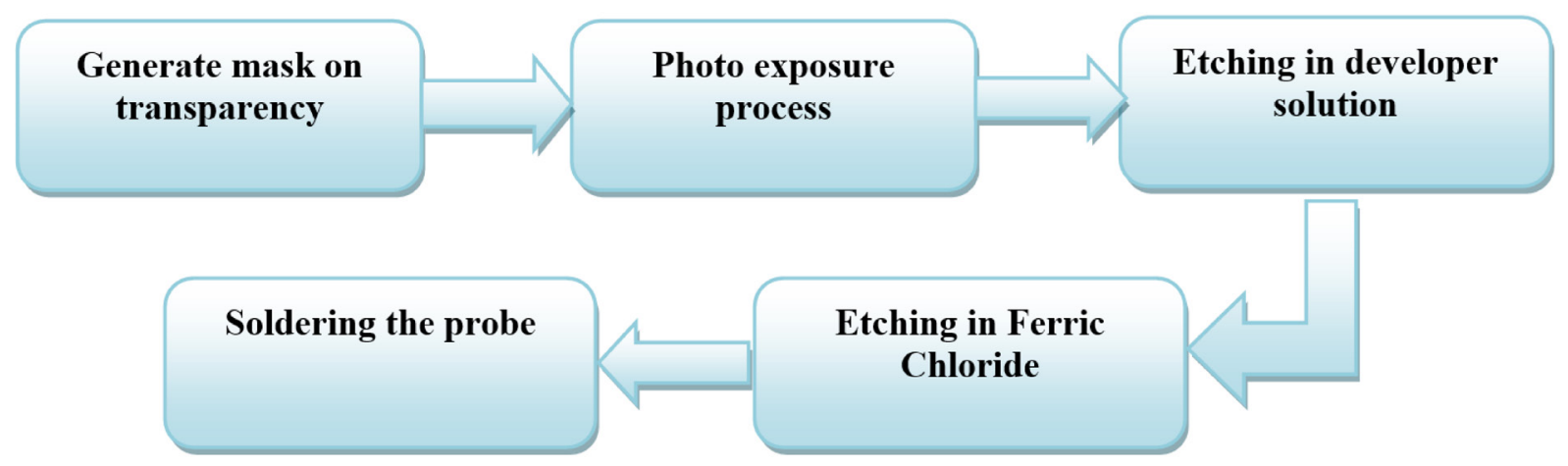

Figure 3: Fabrication process

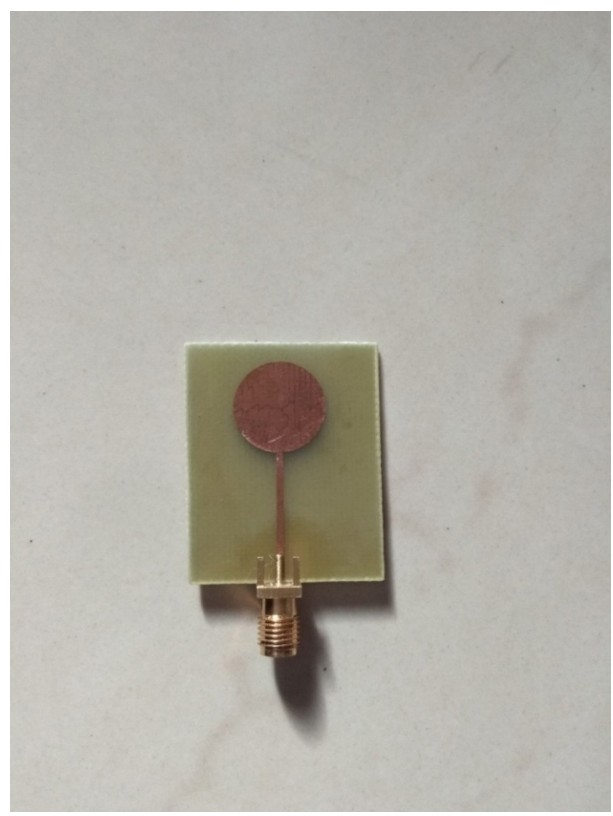

(a)

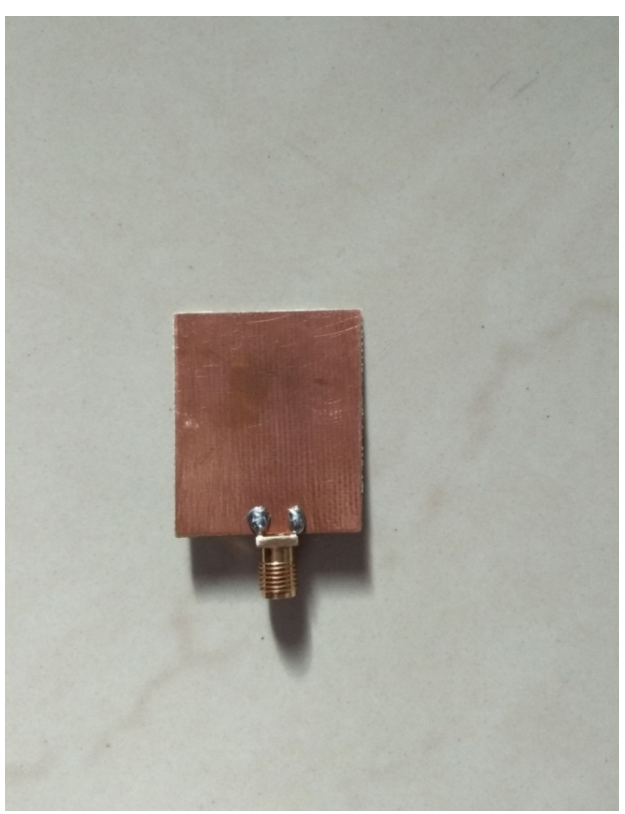

(b)

Figure 4: Patch antenna after fabrication, a) top and b) bottom view 


\section{Results and Discussion}

\subsection{CST Software Simulation Results}

After designing with CST software the circular MSP antenna is shown in Fig. 5.

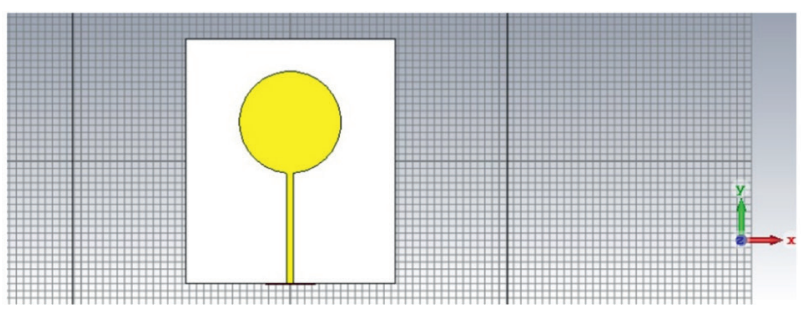

Figure: 5 Circular MSP in CST

1. Balance curve

The Fig. 6 shows the $2 \mathrm{D}$ view of balance curve of simulated MSP. S-parameter of $5.82 \mathrm{GHz}$ is 0.213 which is shown in the Fig. 6.
2. Farfield Directivity

2D radiation pattern and farfield directivity of the antenna is shown in Fig. 7. The Fig. 7 shows maximum gain of the antenna is $6.86 \mathrm{dBi}$ for main lobe and $-8.1 \mathrm{~dB}$ for side lobe at $5.8 \mathrm{GHz}$. The HPBW or half power (3 $\mathrm{dB}$ ) beam width (angular width) is 77.3 degrees.

3. VSWR

The Fig. 10 shows the Voltage Standing Wave Ratio (VSWR) versus frequency graph of the designed antenna. The VSWR is minimum (equal to 1.5414 ) at 5.8 $\mathrm{GHz}$.

\subsection{VNA Results of Fabricated Antenna}

1. Return loss

Return loss of fabricated antenna is $-24.11 \mathrm{~dB}$ which is shown in Fig. 8 with VNA.

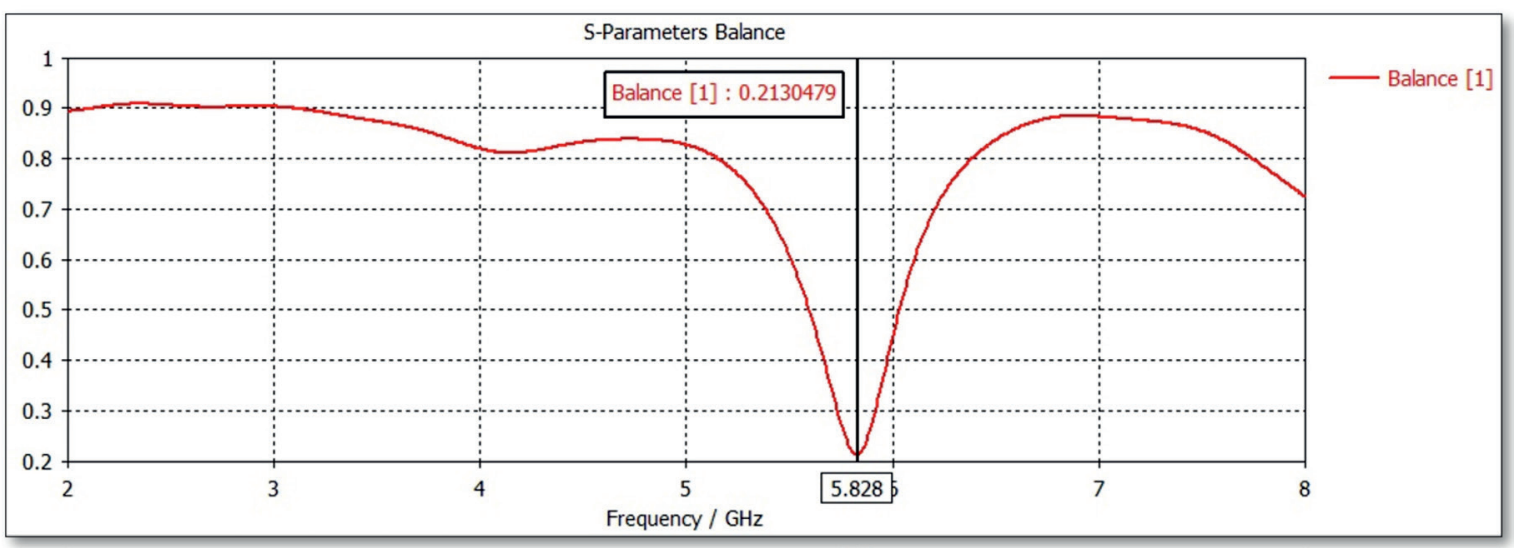

Figure 6: Balance curve of MSP

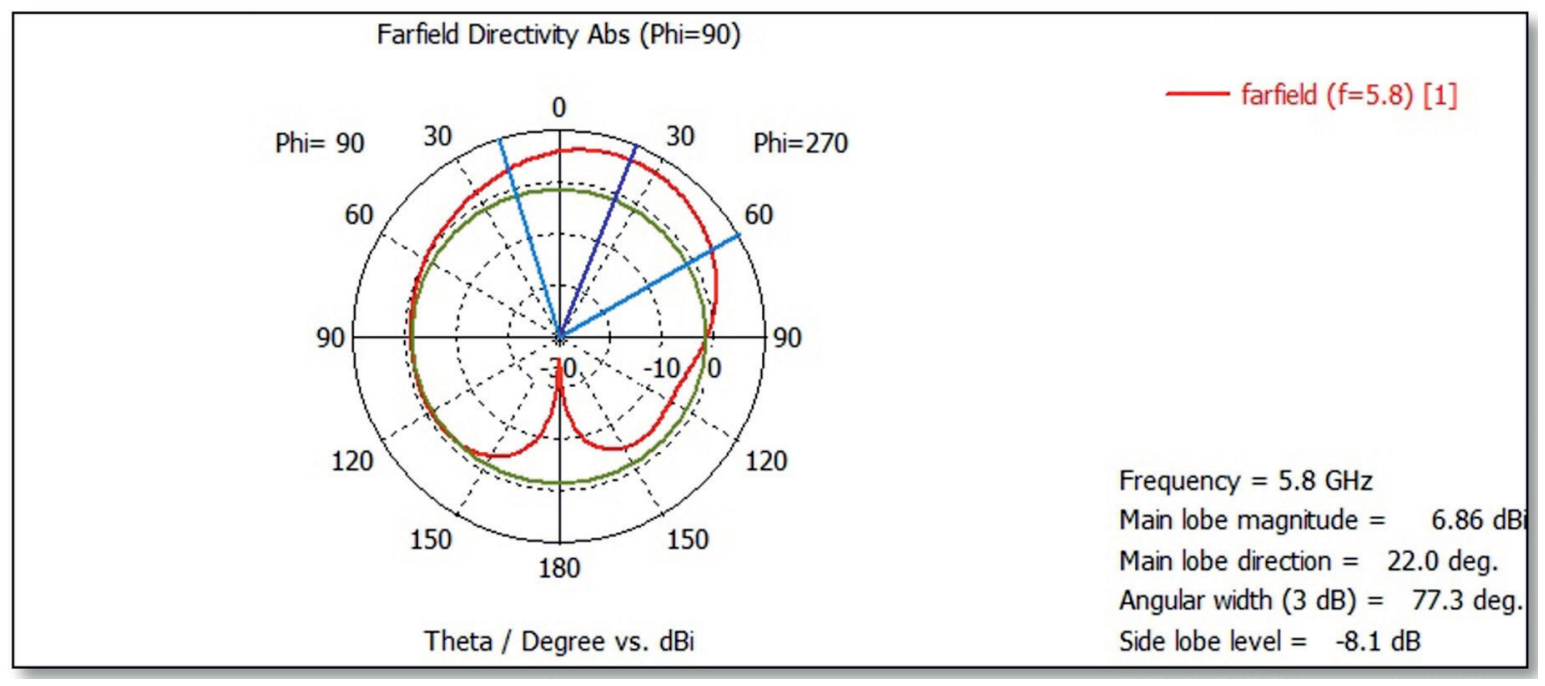

Figure 7: Radiation pattern of MSP 


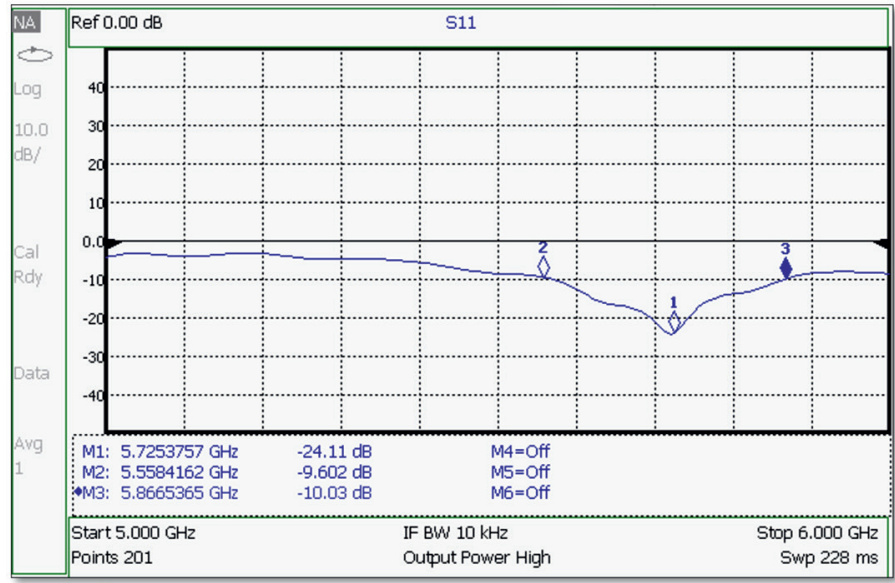

Figure 8: Return loss with VNA

2. Smith Chart

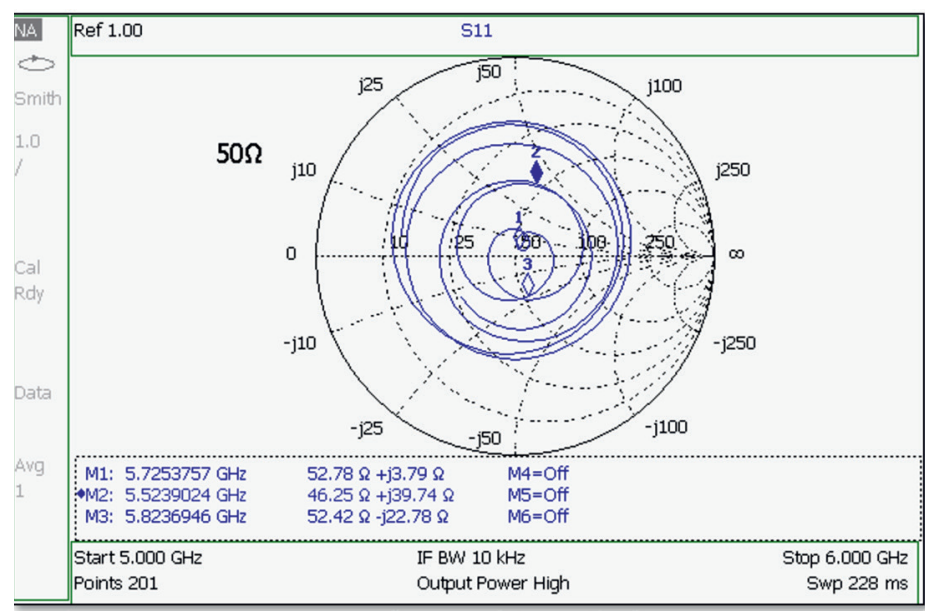

Figure 9: Smith chart of MSP

\section{VSWR}

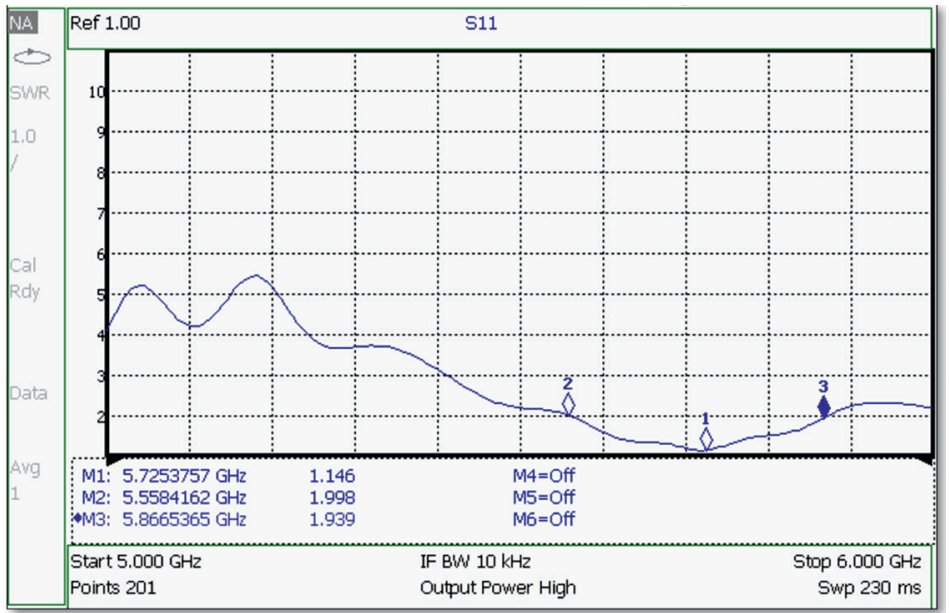

Figure 10: VSWR on VNA for fabricated MSP

VSWR of fabricated MSP is 1.939 at $5.8 \mathrm{GHz}$. 


\section{Conclusion and Discussion}

Antenna designing tools and softwares are very useful for researchers and scientists because, all antenna parameters, responses and results are observed before fabrication. Changes and corrections in the parameters, geometry of antenna can be done before making hardware or real antenna fabrication. Because of the reason, time and money will be saved and also we can found multiple responses with the same geometry. The selection of antenna tools, software's and process of fabrications of any antenna is depending on the antenna elements, geometry and parameters. All antennas designing software's and tools having its own features, specifications and functions with limitations and strength.

The designing and simulation of circular MSP antenna at $5.8 \mathrm{GHz}$ frequency is successfully done with CST software and fabrication of actual patch is also completed. The parameters of simulation patch in the software and actual fabricated patch are same. The VSWR of antenna is 1.54 during simulation on CST and 1.146 on VNA. The return loss of circular patch is less than $-10 d B$.

\section{References}

[1] Wikipedia. Wireless. [Online] Available: https:// en.wikipedia.org/wiki/Wireless [Accessed: 25 August, 2020]

[2] T. B. Tandel and N. Shingala. Design and simulation of microstrip rectangular patch antenna for bluetooth application. International Journal of Latest Technology in Engineering, Management Eे Applied Science, vol. 4, no. 8, pp. 23-27, 2015.

[3] K. Gupta, K. Jain and P. Singh. Analysis and design of circular microstrip patch antenna at $5.8 \mathrm{GHz}$. International Journal of Computer Science and Information Technologies, vol. 5, no. 3, pp. 38953898, 2014.

[4] C.A. Balanis. Antenna theory: Analysis and design. 4th ed. New Jersey, Wiley, 2005.

[5] M. T. I.-u. Huque, M. Chowdhury, M. K. Hosain and M. S. Alam. Performance analysis of corporate feed rectangular patch element and circular patch element 4x2 microstrip array antennas. International Journal of Advanced Computer Science and Applications, vol. 2, no. 7, pp. 74-79, 2011. https://doi.org/10.14569/IJACSA.2011.020711

[6] R. Garg, I, Bahl. Microstrip antenna design handbook. 1st ed. London, Artech House, 2000.

[7] D. Thakur, S. Paul and N. Kalambe. Review of microstrip patch antenna using UWB for wireless communication devices. International Journal of Computer Science and Mobile Computing, vol. 4, no. 1, pp.128-133, 2015.
[8] R. Mishra, R. G. Mishra, R. K. Chaurasia and A. Shrivastava. Design and analysis of microstrip patch antenna for wireless communication. International Journal of Innovative Technology and Exploring Engineering, vol. 8, 2019.

[9] H. Werfelli, K. Tayari, M. Chaoui, M. Lahiani and H. Ghariani. Design of rectangular microstrip patch antenna. 2nd International Conference on Advanced Technologies for Signal and Image Processing - ATSIP'2016, March 21-24, Monastir, Tunisia, 2016.

[10] A.H.M. Z. Alam, Md. R. Islam and S. Khan. Design and analysis of UWB rectangular patch antenna. Pacific conference on applied electromagnetiggs proceedings, December 4- 6, Malaysia, 2007.

[11] W. Houda, M. Chaoui, H. Ghariani and M. Lahiani. Design of a pulse generator for UWB communications. 10th International MultiConferences on Systems Signals \&० Devices 2013 (SSD13), Tunisia, 2013.

[12] K. Kamakshi, A. Singh, M. Aneesh and J. A. Ansari. Novel design of microstrip antenna with improved bandwidth. International Journal of Microwave Science and Technology, vol. 2014, 2014. https://doi.org/10.1155/2014/659592

[13] G. S. Rajput. Design and analysis of rectangular microstrip patch antenna using metamaterial for better efficiency. International Journal of Advanced Technology Eं Engineering Research, vol. 2, no. 6, pp. 51, 2012.

[14] S. S. Khangaro, G. Sujediya, T. Jhajharia and A. Kumar. Design and fabrication of E-SLOT microstrip patch antenna for WLAN application. International Journal of Advanced Research in Computer and Communication Engineering, vol. 4, no. 6, pp. 149-154, 2015.

[15] S. P. Singh, A. Singh, D. Upadhyay, S. Pal and M. Munde. Design and fabrication of microstrip patch antenna at 2.4 Ghz for WLAN application using HFSS. Journal of Electronics and Communication Engineering, pp. 1-6, 2016. https://doi.org/10.9790/2834-150100106

[16] Y. Al. Naiemy, T. A. Elwi, H. R. Khaleel and H. Al. Rizzo. A systematic approach for the design, fabrication, and testing of microstrip antennas using inkjet printing technology. International Scholarly Research Network (ISRN), vol. 2012, 2012. https://doi.org/10.5402/2012/132465

[17] G.B. Hoang, G. N. Van, L.T. Phuong, T. Anh Vu and D. B. Gia. Research, design and fabrication of 2.45 $\mathrm{GHz}$ microstrip patch antenna arrays for closerange Wireless Power Transmission Systems. International Conference on Advanced Technologies for Communications (ATC), 2016. https://doi.org/10.1109/ATC.2016.7764784 


\section{旬}

CHITKARA

UNIVERSITY

\section{Journal on Today's Ideas - Tomorrow's Technologies}

Chitkara University, Saraswati Kendra, SCO 160-161, Sector 9-C, Chandigarh, 160009, India

Volume - 8 , Issue-2

December 2020

ISSN 2321-3906

Copyright: [C 2020 R.V. Shinde et al.] This is an Open Access article published in Journal on Today's Ideas - Tomorrow's Technologies by Chitkara University, Publications. It is published with a Creative Commons Attribution- CC-BY 4.0 International License. This license permits unrestricted use, distribution, and reproduction in any medium, provided the original author and source are credited. 